VOL. 58 (1998) [155-158]

\title{
COMPARABLE CHARACTERISATIONS OF TWO CLASSES OF BANACH SPACES BY SUBDIFFERENTIALS
}

\author{
J.R. GiLeS
}

\begin{abstract}
We characterise Banach spaces not containing $\ell_{1}$ and Banach spaces which are Asplund spaces by continuity properties of the subdifferential mappings of their equivalent norms.
\end{abstract}

Recently, comparable characterisations were given for two classes of Banach spaces in terms of differentiability properties of equivalent norms on the space [2, Theorem 1 , p.265 and Theorem 2, p.268]. Here we reduce these differentiability properties to give comparable weaker sufficiency conditions determining spaces of the two classes.

Theorem 1. A Banach space $X$ does not contain a subspace topologically isomorphic to $\ell_{1}$ if and only if for every equivalent norm $p$ on $X$, given $F \in X^{* *} \backslash\{0\}$ the subdifferential mapping $x \mapsto \partial p(x)$ is Hausdorff $F$-upper semi-continuous and $\partial p(x)$ is $F$-compact at some point $x \in S_{p}(X)$.

THEOREM 2. A Banach space $X$ is an Asplund space if and only if for every equivalent norm $p$ on $X$, the subdifferential mapping $x \mapsto \partial p(x)$ is Hausdorff weak upper semi-continuous and $\partial p(x)$ is weakly compact at some point $x \in S_{p}(X)$.

Given a continuous convex function $\phi$ on an open convex subset $A$ of a Banach space $X$, the subdifferential of $\phi$ at $x \in A$ is the set

$$
\partial \phi(x) \equiv\left\{f \in X^{*}: f(y) \leq \phi_{+}^{\prime}(x)(y) \quad \text { for all } y \in X\right\}
$$

Given the dual $X^{*}$ with a locally convex topology $\tau$, the subdifferential mapping $x \mapsto \partial \phi(x)$ of $A$ into subsets of $X^{*}$ is Hausdorff $\tau$-upper semi-continuous at $x \in A$ if given a $\tau$-neighbourhood $W$ of 0 in $X^{*}$ there exists a $\delta>0$ such that $\partial \phi(y) \subseteq \partial \phi(x)+W$ for all $\|x-y\|<\delta$. The subdifferential mapping is always Hausdorff weak* upper semi-continuous, $[5, \mathrm{p} 19]$. Given $F \in X^{* *} \backslash\{0\}$, by the $F$-topology on $X^{*}$ we mean the locally convex topology induced by the semi-norm $|F|$.

Received 19th January, 1998

Copyright Clearance Centre, Inc. Serial-fee code: 0004-9729/98 \$A2.00+0.00. 
Given a subset $A$ of the dual $X^{*}$ we say that a subset $S$ of $A$ is a weak ${ }^{*}$ exposed subset of $A$ if there exists an $x \in X$ such that $\widehat{x}(S)$ is constant and $\widehat{x}(S)>\widehat{x}(g)$ for all $g \in A \backslash S$. A weak* slice of $A$ by $x \in X$ is a subset of $A$ of the form

$$
S(A, \widehat{x}, \delta) \equiv\{f \in A: f(x)>\sup f(A)-\delta\} \quad \text { for } \delta>0
$$

For the dual $X^{*}$ with a locally convex topology $\tau$ we say that $S$ is a weak ${ }^{*} \tau$-exposed subset of $A$ if it is weak* exposed by some $x \in X$ and given a $\tau$-neighbourhood $W$ of 0 there exists a $\delta>0$ such that $S(A, \widehat{x}, \delta) \subseteq S+W$.

Lemma 1. [3, Theorem 2.1, p.102] Given a Banach space $X$ and dual $X^{*}$ with a locally convex topology $\tau$, the subdifferential mapping $x \mapsto \partial\|x\|$ is Hausdorff $\tau$-upper semi-continuous at $x \in S(X)$ if and only if $\widehat{x}$ weak $^{*} \tau$-exposes the subset $\partial\|x\|$ of $B\left(X^{*}\right)$.

While this result is used to prove Lemma 2, it will be used directly in the proof of Theorem 2. For the proof of Theorem 1, we use the following characterisation.

LEMma 2. [3, Theorem 3.2, p.104] Given a Banach space $X$ and $F \in X^{* *} \backslash\{0\}$, the subdifferential mapping $x \mapsto \partial\|x\|$ is Hausdorff $F$-upper semi-continuous at $x \in$ $S(X)$ and $\partial\|x\|$ is $F$-compact if and only if $F$ restricted $B\left(X^{*}\right)$ is weak* continuous at points of $\partial\|x\|$.

Proof of Theorem 1: One way follows from [2, Theorem 1, (i) $\Longrightarrow$ (v), p.265]. Conversely, it has been shown [1, Corollary 3.3 (ii) $\Longrightarrow$ (i), p.112] that if $X$ contains a subspace topologically isomorphic to $\ell_{1}$ then there exists an $F \in X^{* *} \backslash\{0\}$ and an equivalent norm $p$ on $X$ such that $F$ restricted to $B_{p}\left(X^{*}\right)$ is nowhere weak* continuous.

Lemma 3. A Banach space $X$ is an Asplund space if every non-empty weak* compact convex subset of $X^{*}$ has a weak* weakly exposed subset which is weakly compact.

Proof: Consider $K$ a non-empty closed bounded convex subset of $X^{*}$ and $\overline{c o}^{w^{*}} K$ with weakly compact subset $S$ weak* weakly exposed by $x \in X \backslash\{0\}$. Suppose that $S \cap K=\emptyset$. Then since $S$ is also convex and $K$ is weakly closed they can be strongly separated by a weakly closed hyperplane. Then there exists a weakly open set $W$ such that $S \subseteq W$ and $W \cap K=\emptyset$. Since $S$ is weakly compact there exists a weak open neighbourhood $N$ of 0 such that $S+N \subseteq W$. But since $S$ is a subset of $\overline{c o}^{w^{*}} K$ weak* weakly exposed by $x$ there exists a $\delta>0$ such that $S\left(\overline{c o}^{*} K, \widehat{x}, \delta\right) \subseteq S+N$. But then $(S+N) \cap K \neq \emptyset$, a contradiction. So there exists $f_{0} \in S \cap K$. Now $S$ is an extreme subset of $\overline{c o}^{\omega^{*}} K$ so there exists a nonempty closed convex extreme subset $S_{K}$ of $K$ in $S$. Since $S$ is weakly compact, the Krein-Milman Theorem gives us that $S_{K}$ 
has an extreme point which is then an extreme point of $K$. We conclude that $X^{*}$ has the Krein-Milman property [4] which implies that $X$ is an Asplund space.

Using the duality given in Lemma 1 , we can now proceed.

Proof OF TheOREM 2: One way follows from [2, Theorem 2, (i) $\Longrightarrow$ (v), p.268]. Conversely, suppose there exists a non-empty weak* compact convex subset $A$ of $X^{*}$, (we may suppose $0 \in A$ ), such that given $x \in X \backslash\{0\}$ any subset of $A$ weak* exposed by $x$ is not both weak* weakly exposed and weakly compact. Writing $C \equiv A \cup(-A)$ and $K \equiv C+B\left(X^{*}\right)$ we have that $K$ is weak* compact and convex and any subset of $K$ weak* exposed by $x$ is not both weak* weakly exposed and weakly compact. The equivalent norm $p$ on $X$ defined by $p(x)=\sup \{f(x): f \in K\}$ has $K$ as its corresponding dual ball. So from Lemma 1 we conclude that the subdifferential mapping $x \mapsto \partial p(x)$ is nowhere at points of $S_{p}(X)$ both Hausdorff weakly upper semi-continuous with weakly compact images.

Given a Banach space $X$ and dual $X^{*}$ with a locally convex topology $\tau$, it is instructive to see that the subdifferential mapping $x \mapsto \partial\|x\|$ being Hausdorff $\tau$-upper semi-continuous with $\tau$-compact images has a geometrical characterisation.

Lemma 4. Consider a Banach space $X$ and $x \in S(X)$.

(i) Given $F \in S\left(X^{* *}\right)$, the subdifferential mapping $x \mapsto \partial\|x\|$ is Hausdorff $F$-upper semi-continuous at $x$ if and only if $\widehat{\partial\|x\|}$ is $F$-dense in $\partial\|\widehat{x}\|$.

(ii) The subdifferential mapping $x \mapsto \partial\|x\|$ is Hausdorff weak upper semicontinuous at $x$ if and only if $\widehat{\partial\|x\|}$ is weak ${ }^{*}$ dense in $\partial\|\widehat{x}\|, \overline{[3}$, Theorem 3.1, p.103].

Proof of (i): Suppose that $\widehat{\partial\|x\|}$ is not $F$-dense in $\partial\|\widehat{x}\|$. Then there exists an $\mathcal{F} \in \partial\|\widehat{x}\|$ such that $F$ strongly separates $\mathcal{F}$ from $\widehat{\partial\|x\|}$. Then there exists $r>0$ such that

$$
d(\widehat{F}(\mathcal{F}), \widehat{F}(\widehat{\partial\|x\|}))>r .
$$

Since $B\left(\widehat{X^{*}}\right)$ is weak* dense in $B\left(X^{* * *}\right)$, for each $n \in \mathbb{N}$ there exists $f_{n} \in B\left(X^{*}\right)$ such that

$$
\left|\left(\widehat{f}_{n}-\mathcal{F}\right)(\widehat{x})\right|<\frac{1}{n} \quad \text { and } \quad\left|\left(\widehat{f}_{n}-\mathcal{F}\right)(F)\right|<\frac{1}{n} .
$$

Now $\left|f_{n}(x)-1\right|<1 / n$ for all $n \in \mathbb{N}$. By the Bishop-Phelps Theorem there exist sequences $\left\{y_{n}\right\}$ in $S(X)$ and $\left\{f_{y_{n}}\right\}$ in $S\left(X^{*}\right)$ where $f_{y_{n}} \in \partial\left\|y_{n}\right\|$ such that $\left\{y_{n}\right\}$ is norm convergent to $x$ and $\left\{f_{n}-f_{y_{n}}\right\}$ is norm convergent to 0 . Then since the subdifferential mapping $x \mapsto \partial\|x\|$ is Hausdorff $F$-upper semi-continuous at $x$ we have

$$
d\left(\widehat{F}\left(\widehat{f}_{y_{n}}\right), \widehat{F}(\widehat{\partial\|x\|})\right) \rightarrow 0 \text { as } n \rightarrow \infty
$$


But since $\left|\left(\hat{f}_{n}-\mathcal{F}\right)(F)\right|<1 / n$ for all $n \in \mathbb{N}$ we have contradicted our separation inequality.

Conversely, suppose that $\widehat{\partial\|x\|}$ is $F$-dense in $\partial\|\widehat{x}\|$. Consider an $F$-neighbourhood $W$ of 0 in $X^{*}$. Now $W$ is the restriction to $X^{*}$ of an $F$-neighbourhood $W^{*}$ of 0 in $X^{* * *}$. Since the subdifferential mapping $G \mapsto \partial\|G\|$ is Hausdorff weak* upper semi-continuous on $X^{* *}$, there exists a $\delta>0$ such that

$$
\partial\|\widehat{y}\| \subseteq \partial\|\widehat{x}\|+\frac{W^{*}}{2} \text { for all }\|x-y\|<\delta .
$$

So $\partial\|y\| \subseteq \partial\|x\|+W$ for all $\|x-y\|<\delta$.

Using Lemma 4 we can restate our characterisation theorems as follows:

THEOREM $1^{\prime}$. A Banach space $X$ does not contain a subspace topologically isomorphic to $\ell_{1}$ if and only if for every equivalent norm $p$ on $X$, given $F \in X^{* *} \backslash\{0\}$

$$
F(\partial p(x))=\widehat{F}\left(\partial p^{* *}(\widehat{x})\right)
$$

at some point $x \in S_{p}(X)$.

Theorem $2^{\prime}$. A Banach space $X$ is an Asplund space if and only if for every equivalent norm $p$ on $X$,

$$
\partial \widehat{p(x)}=\partial p^{* *}(\widehat{x})
$$

at some point $x \in S_{p}(X)$.

\section{REFERENCES}

[1] R. Deville, G. Godefroy and V. Zizler, Smoothness and renormings in Banach spaces, Pitman Monographs and Surveys in Pure and Applied Mathematics 64 (Longman Scientific and Technical, Harlow, 1993).

[2] J.R. Giles, 'Comparable differentiability characterisations of two classes of Banach spaces', Bull. Austral. Math. Soc. 56 (1997), 263-272.

[3] J.R. Giles, D.A. Gregory and B. Sims, 'Geometrical implications of upper semi-continuity of the duality mapping on a Banach space', Pacific J. Math. 79 (1978), 99-109.

[4] R.E. Huff and P.D. Morris, 'Dual spaces with the Krein-Milman property have the Radon-Nikodym property', Proc. Amer. Math. Soc. 49 (1975), 104-108.

[5] R.R. Phelps, Convex functions, monotone operators and differentiability (2nd ed.), Lecture Notes in Math. 1364 (Springer-Verlag, Berlin, Heidelberg, New York, 1993). 